\title{
SPECTRA OF SOME ALGEBRAS OF ENTIRE FUNCTIONS OF BOUNDED TYPE, GENERATED BY A SEQUENCE OF POLYNOMIALS
}

\begin{abstract}
In this work, we investigate the properties of the topological algebra of entire functions of bounded type, generated by a countable set of homogeneous polynomials on a complex Banach space.

Let $X$ be a complex Banach space. We consider a subalgebra $H_{b \mathbb{P}}(X)$ of the Fréchet algebra of entire functions of bounded type $H_{b}(X)$, generated by a countable set of algebraically independent homogeneous polynomials $\mathbb{P}$. We show that each term of the Taylor series expansion of entire function, which belongs to the algebra $H_{b \mathbb{P}}(X)$, is an algebraic combination of elements of $\mathbb{P}$. We generalize the theorem for computing the radius function of a linear functional on the case of arbitrary subalgebra of the algebra $H_{b}(X)$ on the space $X$. Every continuous linear multiplicative functional, acting from $H_{b \mathbb{P}}(X)$ to $\mathbb{C}$ is uniquely determined by the sequence of its values on the elements of $\mathbb{P}$. Consequently, there is a bijection between the spectrum (the set of all continuous linear multiplicative functionals) of the algebra $H_{b \mathbb{P}}(X)$ and some set of sequences of complex numbers. We prove the upper estimate for sequences of this set. Also we show that every function that belongs to the algebra $H_{b \mathbb{P}}(X)$, where $X$ is a closed subspace of the space $\ell_{\infty}$ such that $X$ contains the space $c_{00}$, can be uniquely analytically extended to $\ell_{\infty}$ and algebras $H_{b \mathbb{P}}(X)$ and $H_{b \mathbb{P}}(\ell)$ are isometrically isomorphic. We describe the spectrum of the algebra $H_{b \mathbb{P}}(X)$ in this case for some special form of the set $\mathbb{P}$.

Results of the paper can be used for investigations of the algebra of symmetric analytic functions on Banach spaces.

Key words and phrases: $n$-homogeneous polynomial, analytic function, spectrum of algebra.
\end{abstract}

Vasyl Stefanyk Precarpathian National University, 57 Shevchenka str., 76018, Ivano-Frankivsk, Ukraine

E-mail: sv.halushchak@ukr.net

\section{INTRODUCTION}

The theory of analytic functions is an important section of nonlinear functional analysis. In many modern investigations topological algebras of analytic functions and spectra of such algebras are studied.

The existence of algebraic basis plays an important role in the description of the spectrum (the set of all continuous complex-valued linear multiplicative functionals) of the algebra, since every continuous linear multiplicative functional is uniquely defined by its values on elements of the algebraic basis.

The problem of description of spectra of algebras of analytic functions of bounded type was considered by many authors (see, e.g., $[2,3,10])$. In the general case the problem of description of spectra of algebras with the countable algebraic bases is not solved. However for some of

$\mathrm{y} \triangle \mathrm{K} 517.98$

2010 Mathematics Subject Classification: 46J20, 46E15.

The publication contains the results of studies conducted by President's of Ukraine grant for competitive projects 0119U103204. 
these algebras descriptions of spectra were constructed. Algebras of symmetric analytic functions of bounded type on spaces with symmetric structures are typical examples of algebras generated by countable sets of polynomials and were studied in [1,4-8].

We generalize the theorem [2] for computing the radius function of a linear functional on case of arbitrary subalgebra of the Fréchet algebra of entire functions of bounded type $H_{b}(X)$ with the topology of uniform convergence on a complex Banach space $X$. We consider the subalgebra $H_{b \mathbb{P}}(X)$ of the algebra $H_{b}(X)$ of entire functions, generated by a countable set of algebraically independent polynomials $\mathbb{P}=\left\{P_{1}, P_{2}, \ldots, P_{n}, \ldots\right\}$, such that $P_{n}$ is an $n$ homogeneous polynomial for every $n \in \mathbb{N}$. We show that each term of the Taylor series expansion of entire function, which belongs to the algebra $H_{b \mathbb{P}}(X)$, is an algebraic combination of elements of $\mathbb{P}$. Accordingly, every $f \in H_{b \mathbb{P}}(X)$ can be uniquely represented in the form

$$
f(x)=f(0)+\sum_{n=1}^{\infty} \sum_{k_{1}+2 k_{2}+\cdots+n k_{n}=n} a_{k_{1}, k_{2}, \ldots, k_{n}}\left(P_{1}(x)\right)^{k_{1}}\left(P_{2}(x)\right)^{k_{2}} \cdots\left(P_{n}(x)\right)^{k_{n}},
$$

where $x \in X, a_{k_{1}, k_{2}, \ldots, k_{n}} \in \mathbb{C}$ and $k_{1}, k_{2}, \ldots, k_{n}$ are non-negative integers. Therefore every continuous linear multiplicative functional $\varphi$ acting from $H_{b \mathbb{P}}(X)$ to $\mathbb{C}$ is uniquely determined by the sequence $\left(\varphi\left(P_{1}\right), \varphi\left(P_{2}\right), \ldots, \varphi\left(P_{n}\right)\right)$ of its values on elements of $\mathbb{P}$. Consequently, the spectrum of the algebra $H_{b \mathbb{P}}(X)$ is in one-to-one correspondence with some set of sequences of complex numbers. We prove the upper estimate for sequences of this set. Also we show that every function that belongs to the algebra $H_{b \mathbb{P}}(X)$, where $X$ is a closed subspace of the space $\ell_{\infty}$ such that $X$ contains the space $c_{00}$, can be uniquely analytically extended to $\ell_{\infty}$ and algebras $H_{b \mathbb{P}}(X)$ and $H_{b \mathbb{P}}\left(\ell_{\infty}\right)$ are isometrically isomorphic. We describe the spectrum of the algebra $H_{b \mathbb{P}}(X)$ in this case for the set $\mathbb{P}=\left\{P_{1}, P_{2}, \ldots, P_{n}, \ldots\right\}$ such that

$$
P_{n}\left(\left(x_{1}, x_{2}, \ldots, x_{n}, \ldots\right)\right)=x_{n}^{n}
$$

for $n \in \mathbb{N}$.

In the first section we recall some basic notions on the theory of analytic functions on a Banach space and the theory of the Fréchet algebras which are necessary for a full comprehension of the paper.

In the second section we generalize the theorem for computing the radius function of a linear functional on case of arbitrary subalgebra of the Fréchet algebra of entire functions of bounded type $H_{b}(X)$ on a complex Banach space $X$. We also prove that every term of the Taylor series expansion of entire function, generated by the countable set of algebraically independent polynomials, is an algebraic combination of these polynomials.

In the third section of the paper we describe spectra of the Fréchet algebras of entire functions, generated by the sequence of polynomials $\mathbb{P}$ on complex spaces, which are the closed subspaces of the space $\ell_{\infty}$ and contain the linear space $c_{00}$.

\section{PRELIMINARIES}

In this section we will review the formal definition of polynomial on a Banach space and introduce the necessary background. To begin with, we establish some notation. Throughout the whole article the letter $X$ will always stand for a complex Banach space. The set of all positive integers will be denoted by $\mathbb{N}$, whereas the set $\mathbb{N} \cup\{0\}$ will be denoted by $\mathbb{N}_{0}$. We denote 
by $\mathbb{Q}^{+}$the set of all positive rationals. We also denote by $\ell_{\infty}$ the complex Banach space of all bounded sequences $x=\left(x_{1}, x_{2}, \ldots\right)$ of complex numbers with the norm $\|x\|_{\infty}=\sup _{i \in \mathbb{N}}\left|x_{i}\right|$ and by $c_{00}$ the linear space of eventually zero sequences $x=\left(x_{1}, x_{2}, \ldots, x_{n}, 0, \ldots\right)$ of complex numbers with the norm $\|x\|=\sup _{i \in \mathbb{N}}\left|x_{i}\right|$.

Definition 1. For $n \in \mathbb{N}$ a mapping $P: X \rightarrow \mathbb{C}$ is said to be an $n$-homogeneous polynomial if there exists some $n$-linear form $A_{P}: X^{n} \rightarrow \mathbb{C}$ such that $P(x)=A_{P}(\underbrace{x, \ldots, x}_{n})$ for every $x \in X$.

We shall denote by $\mathcal{P}^{n}(X)$ the vector space of all $n$-homogeneous polynomials from $X$ to $\mathbb{C}$. Also let $\mathcal{P}^{0}(X)$ be the vector space of all constant mappings from $X$ to $\mathbb{C}$. For each $P \in \mathcal{P}^{n}(X)$ we shall set

$$
\|P\|=\sup \{|P(x)|: x \in X,\|x\| \leq 1\} .
$$

It is known that a polynomial $P \in \mathcal{P}^{n}(X)$ is continuous if and only if $\|P\|<\infty$.

Definition 2. A mapping $P: X \rightarrow \mathbb{C}$ is said to be a polynomial of degree at most $n$, where $n \in \mathbb{N}_{0}$, if it can be represented as a sum $P=P_{0}+P_{1}+\cdots+P_{n}$, where $P_{j} \in \mathcal{P}^{j}(X)$ for $j=\overline{0, n}$.

Definition 3. Polynomials $P_{1}, P_{2}, \ldots$, where $P_{j} \in \mathcal{P}^{j}(X), j \in \mathbb{N}$, are called algebraically independent polynomials, when for all $n \in \mathbb{N}$ and every polynomial $q: \mathbb{C}^{n} \rightarrow \mathbb{C}$ if the equality $q\left(P_{1}(x), P_{2}(x), \ldots, P_{n}(x)\right)=0$ holds for every $x \in X$, then $q \equiv 0$.

Definition 4. A polynomial $P: X \rightarrow \mathbb{C}$ is called an algebraic combination of elements of $\mathbb{P}=\left\{P_{1}, P_{2}, \ldots\right\}$ if there exists $n \in \mathbb{N}$ and a polynomial $q: \mathbb{C}^{n} \rightarrow \mathbb{C}$ such that $P(x)=$ $q\left(P_{1}(x), \ldots, P_{n}(x)\right)$ for every $x \in X$.

Let us denote by $B(a, r)$ and $\bar{B}(a, r)$ an open and a closed balls of radius $r$ and center $a \in X$ respectively.

Definition 5. Let $U$ be the open subset of $X$. A mapping $f: U \rightarrow \mathbb{C}$ is said to be holomorphic or analytic on $U$ if for each $a \in U$ there exists an open ball $B(a ; r) \subset U$ and a sequence of polynomials $f_{0}, f_{1}, \ldots$, where $f_{0} \in \mathbb{C}$ and $f_{j}$ is a $j$-homogeneous polynomial for each $j \in \mathbb{N}$, such that

$$
f(x)=\sum_{n=0}^{\infty} f_{n}(x-a)
$$

uniformly for $x \in B(a ; r)$.

Note that the power series $\sum_{n=0}^{\infty} f_{n}(x-a)$ is called the Taylor series of the function $f$ at the point $a$. If $U=X$ the function $f$ is called an entire function.

According to [9, p. 47, Corollary 7.3] the terms of the Taylor series of an entire function $f: X \rightarrow \mathbb{C}$ can be found using the Cauchy's integral formula

$$
f_{n}(x)=\frac{1}{2 \pi i} \int_{|\zeta|=r} \frac{f(\zeta x)}{\zeta^{n+1}} d \zeta, \quad \text { where } r>0 .
$$

Also recall that the radius of convergence $\rho_{a}$ of the power series $\sum_{n=0}^{\infty} f_{n}(x-a)$ is the supremum of all $r \geq 0$ such that the series converges uniformly on the ball $\bar{B}(a, r)$. According to [4, p. 27, Theorem 4.3], the radius of convergence of the power series is given by the CauchyHadamard formula

$$
\frac{1}{\rho_{a}}=\limsup _{n \rightarrow \infty}\left\|f_{n}\right\|_{1}^{\frac{1}{n}}
$$


Denote by $H_{b}(X)$ the algebra of $\mathbb{C}$-valued entire functions of bounded type on $X$, that is, the space of all entire mappings from $X$ to $\mathbb{C}$, which are bounded on bounded subsets. We endow the algebra $H_{b}(X)$ with the system of uniform norms

$$
\|f\|_{r}=\sup \{|f(x)|: x \in X,\|x\|<r\}, \quad \text { where } r \in \mathbb{Q}^{+} .
$$

It is known that the topology on a countably-normed space can be given by some metric. Note that the algebra $H_{b}(X)$ is complete with respect to this metric. Hence $H_{b}(X)$ is a Frechet algebra.

Let $H_{b}^{(0)}(X)$ be an arbitrary subalgebra of $H_{b}(X)$. For every continuous linear functional $\varphi \in\left(H_{b}^{(0)}(X)\right)^{*}$ there exists $r \in \mathbb{Q}^{+}$such that $\varphi$ is continuous with respect to the norm $\|\cdot\|_{r}$, where $\left(H_{b}^{(0)}(X)\right)^{*}$ is the space of all continuous linear functionals on $H_{b}^{(0)}(X)$.

Analogically to [2, Section 2] let us define the radius function on $\left(H_{b}^{(0)}(X)\right)^{*}$ as follows.

Definition 6. For $\varphi \in\left(H_{b}^{(0)}(X)\right)^{*}$ let the radius function $R(\varphi)$ be the infimum of all $r>0$ such that $\varphi$ is continuous with respect to the norm $\|\cdot\|_{r}$.

Thus,

$$
0 \leq R(\varphi)<\infty .
$$

For $n \in \mathbb{N}_{0}$ let $\widetilde{\mathcal{P}^{n}}(X)=\mathcal{P}^{n}(X) \cap H_{b}^{(0)}(X)$ denote the space of $n$-homogeneous polynomials on $X$, which belong to $H_{b}^{(0)}(X)$. For each $P \in \widetilde{\mathcal{P}^{n}}(X)$ we shall set

$$
\|P\|=\|P\|_{1}=\sup \{|P(x)|: x \in X,\|x\| \leq 1\} .
$$

Each $f \in H_{b}^{(0)}(X)$ has a Taylor series expansion

$$
f=\sum_{n=0}^{\infty} f_{n}
$$

where $f_{n} \in \widetilde{\mathcal{P}^{n}}(X)$ for $n \in \mathbb{N}_{0}$, and the series (2) converges in $H_{b}^{(0)}(X)$, that is, the series (2) converges uniformly to $f$ on each bounded subset of $X$.

Let $\varphi \in\left(H_{b}^{(0)}(X)\right)^{*}$. Taking into account the continuity and the linearity of $\varphi$, we obtain

$$
\varphi(f)=\sum_{n=0}^{\infty} \varphi\left(f_{n}\right)
$$

We denote by $\varphi_{n}$ the restriction of $\varphi \in\left(H_{b}^{(0)}(X)\right)^{*}$ to $\widetilde{\mathcal{P}^{n}}(X)$. Then $\varphi_{n}$ is continuous. Its norm on $\widetilde{\mathcal{P}^{n}}(X)$ will be denoted by

$$
\left\|\varphi_{n}\right\|=\sup \left\{|\varphi(P)|: P \in \widetilde{\mathcal{P}^{n}}(X),\|P\| \leq 1\right\} .
$$

Definition 7. The spectrum of the topological algebra $A$ is the set of all continuous complexvalued linear multiplicative functionals $=$ continuous complex-valued homomorphisms $=$ continuous characters. 


\section{Algebras, GeNERATED By A COUNTABle SET OF POLYNOMiAls}

Let $\mathcal{P}_{\mathbb{P}}(X)$ be the algebra, consisting of all polynomials, which are algebraic combinations of elements of the set $\mathbb{P}$. Let us denote by $H_{b \mathbb{P}}(X)$ the closure of $\mathcal{P}_{\mathbb{P}}(X)$ in the metric of the algebra $H_{b}(X)$. It can be checked that $H_{b \mathbb{P}}(X)$ is a subalgebra of $H_{b}(X)$ and that $H_{b \mathbb{P}}(X)$ is a Frechet algebra with respect to the metric of the algebra $H_{b}(X)$.

Proposition 1. Each term of the Taylor series of a function $f \in H_{b \mathbb{P}}(X)$ can be uniquely represented as an algebraic combination of elements of the set $\mathbb{P}$. Consequently,

$$
f(x)=f(0)+\sum_{n=1}^{\infty} \sum_{k_{1}+2 k_{2}+\cdots+n k_{n}=n} a_{k_{1}, k_{2}, \ldots, k_{n}}\left(P_{1}(x)\right)^{k_{1}}\left(P_{2}(x)\right)^{k_{2}} \cdots\left(P_{n}(x)\right)^{k_{n}},
$$

where $x \in X, a_{k_{1}, k_{2}, \ldots, k_{n}} \in \mathbb{C}$ and $k_{1}, k_{2} \ldots, k_{n} \in \mathbb{N}_{0}$.

Proof. For $n \in \mathbb{N}$ let $f_{n}$ be the $n$th term of the Taylor series of $f$. Let us show that $f_{n}$ can be uniquely represented as an algebraic combination of polynomials $P_{1}, \ldots, P_{n}$.

Let us denote by $\mathcal{P}_{\mathbb{P}_{n}}^{n}(X)$ the space of all $n$-homogeneous polynomials, which are algebraic combinations of polynomials $P_{1}, \ldots, P_{n}$. Note that the set of polynomials of the form $P_{1}^{k_{1}} P_{2}^{k_{2}} \ldots P_{n}^{k_{n}}$, where $k_{1}, k_{2}, \ldots, k_{n} \in \mathbb{N}_{0}$ and $k_{1}+2 k_{2}+\ldots+n k_{n}=n$, is a Hamel basis for the space $\mathcal{P}_{\mathbb{P}_{n}}^{n}(X)$. Since there is a finite number of such polynomials the space $\mathcal{P}_{\mathbb{P}_{n}}^{n}(X)$ is a finitedimensional. Therefore $\mathcal{P}_{\mathbb{P}_{n}}^{n}(X)$ is complete with respect to each of the norms. In particular $\mathcal{P}_{\mathbb{P}_{n}}^{n}(X)$ is complete with respect to the norm $\|\cdot\|_{1}$.

Since $H_{b \mathbb{P}}(X)$ is the closure of the algebra $\mathcal{P}_{\mathbb{P}}(X)$, then there exists a sequence $\left\{a_{l}\right\}_{l=1}^{\infty} \subset$ $\mathcal{P}_{\mathbb{P}}(X)$, which converges to the function $f$ with respect to the metric of $H_{b}(X)$. Let $a_{l n}$ be the $n$th member of the Taylor series of the polynomial $a_{l}$. Note that $a_{l n} \in \mathcal{P}_{\mathbb{P}_{n}}^{n}(X)$ for each $l \in \mathbb{N}$. Let us show that the sequence $\left\{a_{l n}\right\}_{l=1}^{\infty}$ converges to $f_{n}$ with respect to the norm $\|\cdot\|_{1}$. According to the Cauchy's integral formula (1), in which we take $r=1$,

$$
f_{n}(x)=\frac{1}{2 \pi i} \int_{|\zeta|=1} \frac{f(\zeta x)}{\zeta^{n+1}} d \zeta \quad \text { and } \quad a_{l n}(x)=\frac{1}{2 \pi i} \int_{|\zeta|=1} \frac{a_{l}(\zeta x)}{\zeta^{n+1}} d \zeta .
$$

Therefore

$$
\begin{aligned}
\left|f_{n}(x)-a_{l n}(x)\right| & =\left|\frac{1}{2 \pi i} \int_{|\zeta|=1} \frac{f(\zeta x)-a_{l}(\zeta x)}{\zeta^{n+1}} d \zeta\right| \\
& \leq \frac{1}{2 \pi} \int_{|\zeta|=1} \frac{\left|f(\zeta x)-a_{l}(\zeta x)\right|}{|\zeta|^{n+1}} d \zeta=\frac{1}{2 \pi} \int_{|\zeta|=1}\left|f(\zeta x)-a_{l}(\zeta x)\right| d \zeta .
\end{aligned}
$$

When $x \in X$ is such that $\|x\| \leq 1$ and $\zeta \in \mathbb{C}$ is such that $|\zeta|=1$, we obtain $\|\zeta x\| \leq 1$. So when $\|x\| \leq 1$ we have

$$
\left|f(\zeta x)-a_{l}(\zeta x)\right| \leq\left\|f-a_{l}\right\|_{1}
$$

It follows that

$$
\left\|f_{n}-a_{l n}\right\|_{1}=\sup _{\|x\| \leq 1}\left|f_{n}(x)-a_{l n}(x)\right| \leq \frac{1}{2 \pi}\left\|f-a_{l}\right\|_{1} \int_{|\zeta|=1} d \zeta=\left\|f-a_{l}\right\|_{1} .
$$

Since $a_{l} \rightarrow f$ as $l \rightarrow \infty$, then $\left\|f-a_{l}\right\|_{1} \rightarrow 0$ as $l \rightarrow \infty$. Therefore $\left\|f_{n}-a_{l n}\right\|_{1} \rightarrow 0$ as $l \rightarrow \infty$. Hence $a_{l n} \rightarrow f_{n}$ as $l \rightarrow \infty$ with respect to the norm $\|\cdot\|_{1}$. Since $\mathcal{P}_{\mathbb{P}_{n}}^{n}(X)$ is complete with respect to the norm $\|\cdot\|_{1}$, then $f_{n} \in \mathcal{P}_{\mathbb{P}_{n}}^{n}(X)$. Hence $f_{n}$ can be represented as an algebraic combination of polynomials $P_{1}, P_{2}, \ldots, P_{n}$. Such representation is unique, since polynomials $P_{1}, P_{2}, \ldots, P_{n}$ are algebraic independent. 
Let $M_{b \mathbb{P}}$ be the spectrum of the algebra $H_{b \mathbb{P}}(X)$. According to the Proposition 1 every function $f \in H_{b \mathbb{P}}(X)$ can be uniquely represented in the form

$$
f=\sum_{n=0}^{\infty} f_{n}=f(0)+\sum_{n=1}^{\infty} \sum_{k_{1}+2 k_{2}+\ldots+n k_{n}=n} a_{k_{1}, k_{2} \ldots, k_{n}} P_{1}^{k_{1}} P_{2}^{k_{2}} \cdots P_{n}^{k_{n}},
$$

where $a_{k_{1}, k_{2} \ldots, k_{n}} \in \mathbb{C}$ and $k_{1}, k_{2} \ldots, k_{n} \in \mathbb{N}_{0}$. Consequently, for every non-trivial character $\varphi \in$ $M_{b \mathbb{P}}$, taking into account that $\varphi(1)=1$, we have the following:

$$
\varphi(f)=f(0)+\sum_{n=1}^{\infty} \sum_{k_{1}+2 k_{2}+\ldots+n k_{n}=n} a_{k_{1}, k_{2} \ldots, k_{n}}\left(\varphi\left(P_{1}\right)\right)^{k_{1}}\left(\varphi\left(P_{2}\right)\right)^{k_{2}} \cdots\left(\varphi\left(P_{n}\right)\right)^{k_{n}} .
$$

Thus we can see that $\varphi$ is completely defined by its values on polynomials $P_{j}$, where $j \in \mathbb{N}$. Hence we can identify every $\varphi \in M_{b \mathbb{P}}$ with the sequence $\left\{\varphi\left(P_{j}\right)\right\}_{j=1}^{\infty}$.

Let us prove the following analog of [2, Theorem 2.3] on case of arbitrary subalgebra $H_{b}^{(0)}(X)$ of algebra $H_{b}(X)$. Let us recall that we denote by $\varphi_{n}$ the restriction of $\varphi \in\left(H_{b}^{(0)}(X)\right)^{*}$ to $\widetilde{\mathcal{P}^{n}}(X)$, where $\widetilde{\mathcal{P}^{n}}(X)=\mathcal{P}^{n}(X) \cap H_{b}^{(0)}(X)$.

Theorem 1. The radius function $R$ on $\left(H_{b}^{(0)}(X)\right)^{*}$ is given by

$$
R(\varphi)=\limsup _{n \rightarrow \infty}\left\|\varphi_{n}\right\|^{\frac{1}{n}}
$$

Proof. Suppose that $0<t<\limsup _{n \rightarrow \infty}\left\|\varphi_{n}\right\|^{\frac{1}{n}}$. Then there is a sequence of homogeneous polynomials $P_{j}$ of degree $n_{j} \rightarrow \infty$ such that $\left\|P_{j}\right\|=1$ and $\left|\varphi\left(P_{j}\right)\right|>t^{n_{j}}$. If $0<r<t$, then by homogeneity, $\left\|P_{j}\right\|_{r}=r^{n_{j}}$, so that

$$
\left|\varphi\left(P_{j}\right)\right|>\left(\frac{t}{r}\right)^{n_{j}}\left\|P_{j}\right\|_{r}
$$

and $\varphi$ is not continuous with respect to the norm of uniform convergence on $r B$. It follows that $R(\varphi) \geq r$, and on account of the arbitrary choices of $r$ and $t$ we obtain $R(\varphi) \geq \limsup _{n \rightarrow \infty}\left\|\varphi_{n}\right\|^{\frac{1}{n}}$.

For the reverse inequality, let $s$ be strictly larger than the supremum above, so that $\left\|\varphi_{n}\right\| \leq s^{n}$ for $n$ large. Then there is $c \geq 1$ such that $\left\|\varphi_{n}\right\| \leq c s^{n}, n \geq 0$. Let $r>s$ is arbitrary, and a function $f \in H_{b}^{(0)}$ has Taylor series (2). Then the Cauchy estimates yield

$$
r^{n}\left\|f_{n}\right\|=\left\|f_{n}\right\|_{r} \leq\|f\|_{r}, n \geq 0 .
$$

Hence, $\left|\varphi\left(f_{n}\right)\right| \leq\left\|\varphi_{n}\right\|\left\|f_{n}\right\| \leq \frac{c s^{n}}{r^{n}}\|f\|_{r}$, so that in view of (3) we obtain

$$
|\varphi(f)| \leq c\left(\sum_{n=0}^{\infty} \frac{s^{n}}{r^{n}}\right)\|f\|_{r} .
$$

Thus $\varphi$ is continuous with respect to the norm of uniform convergence on the ball $r B$ and $R(\varphi) \leq r$. On account of the arbitrary choices of $s$ and $r$, we can see that

$$
R(\varphi) \leq \limsup _{n \rightarrow \infty}\left\|\varphi_{n}\right\|^{\frac{1}{n}}
$$


Proposition 2. For every $\varphi \in M_{b \mathbb{P}}$ there exists $r \in \mathbb{Q}^{+}$, such that the estimate

$$
\left|\varphi\left(P_{n}\right)\right| \leq r^{n}\left\|P_{n}\right\|_{1}
$$

holds for all $P_{n} \in \mathcal{P}_{\mathbb{P}_{n}}^{n}(X)$.

Proof. Each $\varphi \in M_{b \mathbb{P}}$ is continuous with respect to the norm of uniform convergence on some ball in $X$. Let $\varphi$ be continuous with respect to the norm $\|\cdot\|_{r}$, where $r \in \mathbb{Q}^{+}$. Since the norm of every non-trivial continuous complex valued homomorphism is equal to 1 , the estimate

$$
\left|\varphi\left(P_{n}\right)\right| \leq\left\|P_{n}\right\|_{r}, P_{n} \in \mathcal{P}_{\mathbb{P}_{n}}^{n}(X)
$$

holds for all $r>R(\varphi)$.

So, $\left|\varphi\left(P_{n}\right)\right| \leq \sup _{\|x\|<r}\left|P_{n}(x)\right|$. Let us make the following replacement $x=r y$. Thus we obtain $\left|\varphi\left(P_{n}\right)\right| \leq r^{n} \sup _{n}\left|P_{n}(y)\right|,\left|\varphi\left(P_{n}\right)\right| \leq r^{n}\left\|P_{n}\right\|_{1}$.

$\|y\| \leq 1$

\section{The Case of Subspace $X, c_{00} \subset X \subset \ell_{\infty}$}

Let $X$ be a closed subspace of $\ell_{\infty}$ such that $X$ contains $c_{00}$ and $\mathbb{P}$ be a sequence of continuous polynomials $P_{1}, \ldots, P_{n}, \ldots$ such that

1. $P_{n}$ is an $n$-homogeneous polynomial;

2. $P_{n}$ 's are algebraically independent;

3. every $P_{n}$ depends only on a finite number of coordinates.

Lemma 1. Let us define the mapping $J: H_{b \mathbb{P}}\left(\ell_{\infty}\right) \rightarrow H_{b \mathbb{P}}(X)$ by $J(g)=\left.g\right|_{X}$, where $g \in$ $H_{b \mathbb{P}}\left(\ell_{\infty}\right)$. Let $g_{n} \in H_{b \mathbb{P}}\left(\ell_{\infty}\right)$ be an $n$-homogeneous polynomial. Then the following equality holds:

$$
\left\|g_{n}\right\|_{1}=\left\|J\left(g_{n}\right)\right\|_{1} .
$$

Proof. According to the Proposition 1 each term of the Taylor series $g_{n}$ can be uniquely represented as an algebraic combination of polynomials $P_{1}, \ldots, P_{n}$. Since in our case every polynomial $P_{n}$ depends on a finite number of coordinates, then polynomials $g_{n}$ depend on a finite number of variables. Let us denote by $\kappa(j)$ the maximum among indices of elements of the sequence $x$ on which the polynomial $P_{j}$ depends on, $j=\overline{1, n}, n \in \mathbb{N}$. Obviously, $\kappa(j) \in \mathbb{N}$. Also let us denote by $\kappa_{\max }=\max \{\kappa(j): 1 \leq j \leq n\}$. Then we can write down the following chain of equalities:

$$
\begin{aligned}
\left\|g_{n}\right\|_{1} & =\sup \left\{\left|g_{n}(x)\right|: x=\left(x_{1}, \ldots, x_{m}, \ldots\right) \in \ell_{\infty}, x_{m}=0 \forall m>\kappa_{\max }, m \in \mathbb{N},\|x\| \leq 1\right\} \\
& =\sup \left\{\left|g_{n}(x)\right|: x \in c_{00},\|x\| \leq 1\right\} .
\end{aligned}
$$

Thinking analogically we obtain the following chain of equalities for norms of $J\left(g_{n}\right) \in H_{b \mathbb{P}}(X)$ :

$$
\begin{aligned}
\left\|J\left(g_{n}\right)\right\|_{1} & =\sup \left\{\left|J\left(g_{n}(x)\right)\right|: x=\left(x_{1}, \ldots, x_{m}, \ldots\right) \in X,\|x\| \leq 1\right\} \\
& =\sup \left\{\left|g_{n}(x)\right|: x=\left(x_{1}, \ldots, x_{m}, \ldots\right) \in X,\|x\| \leq 1\right\} \\
& =\sup \left\{\left|g_{n}(x)\right|: x=\left(x_{1}, \ldots, x_{m}, \ldots\right) \in X, x_{m}=0 \forall m>\kappa_{\text {max }}, m \in \mathbb{N},\|x\| \leq 1\right\} \\
& =\sup \left\{\left|g_{n}(x)\right|: x \in c_{00},\|x\| \leq 1\right\} .
\end{aligned}
$$

Thus the equality $\left\|g_{n}\right\|_{1}=\left\|J\left(g_{n}\right)\right\|_{1}$ is established. 
Theorem 2. Every function that belongs to $H_{b \mathbb{P}}(X)$ can be uniquely analytically extended to $\ell_{\infty}$ and algebras $H_{b \mathbb{P}}(X)$ and $H_{b \mathbb{P}}\left(\ell_{\infty}\right)$ are isometrically isomorphic.

Proof. Let us consider a mapping $J: H_{b \mathbb{P}}\left(\ell_{\infty}\right) \rightarrow H_{b \mathbb{P}}(X)$ such that $J(f)=\left.f\right|_{X}$ for every function $f \in H_{b \mathbb{P}}\left(\ell_{\infty}\right)$. It is easy to check that $J$ is a homomorphism from $H_{b \mathbb{P}}\left(\ell_{\infty}\right)$ onto $H_{b \mathbb{P}}(X)$.

Next we will show that the mapping $J$ is a bijection. Firstly, let us prove that for all $f_{1}, f_{2} \in$ $H_{b \mathbb{P}}\left(\ell_{\infty}\right)$ whenever $J\left(f_{1}\right)=J\left(f_{2}\right)$, then $f_{1}=f_{2}$, that is $J$ is an injection. Let us consider $g \in$ $H_{b \mathbb{P}}\left(\ell_{\infty}\right)$ such that $g=f_{1}-f_{2}$ and $g$ has a Taylor series representation $g=\sum_{n=0}^{\infty} g_{n}$. By assumption, $J\left(f_{1}\right)=J\left(f_{2}\right)$, and so $J(g)=J\left(f_{1}-f_{2}\right)=J\left(f_{1}\right)-J\left(f_{2}\right)=0$. On the other hand,

$$
J(g)=J\left(\sum_{n=0}^{\infty} g_{n}\right)=\sum_{n=0}^{\infty} J\left(g_{n}\right)
$$

and the Cauchy estimate yields $\left\|J\left(g_{n}\right)\right\|_{1} \leq\|J(g)\|_{1}, n \in \mathbb{N}_{0}$. Since $J(g)=0$, then $\|J(g)\|_{1}=0$ and $\left\|J\left(g_{n}\right)\right\|_{1}=0$. According to Lemma 1 we have $\left\|g_{n}\right\|_{1}=\left\|J\left(g_{n}\right)\right\|_{1}$. Therefore $\left\|g_{n}\right\|_{1}=0$ and it follows that $g_{n}(x)=0$ for all $x \in \ell_{\infty}$.

Thus we obtain the chain of equalities

$$
f_{1}(x)-f_{2}(x)=g(x)=\sum_{n=0}^{\infty} g_{n}(x)=0
$$

for all $x \in \ell_{\infty}$. It follows that $f_{1}=f_{2}$. Hence, the mapping $J$ is injective.

Now let us show that $J$ is a surjection, that is for every $h \in H_{b \mathbb{P}}(X)$ there is at least one $\widetilde{h} \in H_{b \mathbb{P}}\left(\ell_{\infty}\right)$, such that $J(\widetilde{h})=h$. Since $h \in H_{b \mathbb{P}}(X)$, it has a Taylor series representation $h=\sum_{n=0}^{\infty} h_{n}$ with the radius of convergence

$$
\rho_{0}(h)=\frac{1}{\limsup _{n \rightarrow \infty}\left\|h_{n}\right\|_{1}^{\frac{1}{n}}}=\infty
$$

for all $x \in X$. Let us show that the last equality also holds for all $x \in \ell_{\infty}$. According to Proposition 1 each term $h_{n}$ of the Taylor series of $h$ can be uniquely represented as an algebraic combination of polynomials $P_{1}, \ldots, P_{n}$. Since every polynomial $P_{n}$ depends on a finite number of coordinates, then polynomials $h_{n}$ depend on a finite number of variables. Let us denote by $\kappa(j)$ the maximum among indices of elements of the sequence $x$ on which the polynomial $P_{j}$ depends on, $j=\overline{1, n}, n \in \mathbb{N}$. Obviously, $\kappa(j) \in \mathbb{N}$. Also let us denote by $\kappa_{\max }=\max \{\kappa(j): 1 \leq j \leq n\}$. Then we have the following chain of equalities

$$
\begin{aligned}
\left\|h_{n}\right\|_{1} & =\sup \left\{\left|h_{n}(x)\right|: x=\left(x_{1}, \ldots, x_{k}, \ldots\right) \in X,\|x\| \leq 1\right\} \\
& =\sup \left\{\left|h_{n}(x)\right|: x=\left(x_{1}, \ldots, x_{k}, \ldots\right) \in X, x_{k}=0 \forall k>\kappa_{\text {max }}, k \in \mathbb{N},\|x\| \leq 1\right\} \\
& =\sup \left\{\left|h_{n}(x)\right|: x \in c_{00},\|x\| \leq 1\right\} \\
& =\sup \left\{\left|h_{n}(x)\right|: x=\left(x_{1}, \ldots, x_{k}, \ldots\right) \in \ell_{\infty}, x_{k}=0 \forall k>\kappa_{\text {max }}, k \in \mathbb{N},\|x\| \leq 1\right\} \\
& =\sup \left\{\left|h_{n}(x)\right|: x=\left(x_{1}, \ldots, x_{k}, \ldots\right) \in \ell_{\infty},\|x\| \leq 1\right\}=\left\|\widetilde{h}_{n}\right\|_{1} .
\end{aligned}
$$

Therefore $\lim \sup _{n \rightarrow \infty}\left\|\widetilde{h}_{n}\right\|_{1}^{\frac{1}{n}}=0$ and respectively $\rho_{0}(\widetilde{h})=\frac{1}{\limsup _{n \rightarrow \infty}\left\|\widetilde{h}_{n}\right\|_{1}^{\frac{1}{n}}}=\infty$ for all $x \in \ell_{\infty}$. Hence every function $h \in H_{b \mathbb{P}}(X)$ can be uniquely analytically extended to $\ell_{\infty}$. This extension is a desired function $\widetilde{h}$. Thus the mapping $J$ is surjective. 
It remains to prove that the given function $J$ is an isometry between algebras $H_{b \mathbb{P}}\left(\ell_{\infty}\right)$ and $H_{b \mathbb{P}}(X)$. For this it is sufficient to show that for all $h \in H_{b \mathbb{P}}(X), \widetilde{h} \in H_{b \mathbb{P}}\left(\ell_{\infty}\right)$ such that $J(\widetilde{h})=$ $h$, and $r \in \mathbb{Q}^{+}$the following equality $\|J(\widetilde{h})\|_{r}=\|\widetilde{h}\|_{r}$ holds, that is $\|h\|_{r}=\|\widetilde{h}\|_{r}$.

Let $h=\sum_{n=0}^{\infty} h_{n}$ and $\widetilde{h}=\sum_{n=0}^{\infty} \widetilde{h}_{n}$ be the Taylor series representations of the functions $h \in$ $H_{b \mathbb{P}}(X)$ and $\widetilde{h} \in H_{b \mathbb{P}}\left(\ell_{\infty}\right)$ respectively. Also let $S_{n+1}=h_{0}+\ldots+h_{n}$ and $\widetilde{S}_{n+1}=\widetilde{h}_{0}+\ldots+\widetilde{h}_{n}$ be the partial sums of the given Taylor series. Then the following equalities hold:

$$
\begin{aligned}
& \lim _{n \rightarrow \infty}\left\|h-S_{n+1}\right\|_{r}=0, \\
& \lim _{n \rightarrow \infty}\left\|\widetilde{h}-\widetilde{S}_{n+1}\right\|_{r}=0 .
\end{aligned}
$$

Besides, by the continuity of a norm we have the following inequalities:

$$
\begin{gathered}
\left|\|h\|_{r}-\left\|S_{n+1}\right\|_{r}\right| \leq\left\|h-S_{n+1}\right\|_{r}, \\
\left|\|\widetilde{h}\|_{r}-\left\|\widetilde{S}_{n+1}\right\|_{r}\right| \leq\left\|\widetilde{h}-\widetilde{S}_{n+1}\right\|_{r} .
\end{gathered}
$$

Taking into account (4) and (6) we obtain

$$
\lim _{n \rightarrow \infty}\left\|S_{n+1}\right\|_{r}=\|h\|_{r}
$$

Analogically, by (5) and (7) we have

$$
\lim _{n \rightarrow \infty}\left\|\widetilde{S}_{n+1}\right\|_{r}=\|\widetilde{h}\|_{r}
$$

Therefore $\lim _{n \rightarrow \infty}\left\|S_{n+1}\right\|_{r}=\lim _{n \rightarrow \infty}\left\|\widetilde{S}_{n+1}\right\|_{r}$ and so $\|h\|_{r}=\|\widetilde{h}\|_{r}$. Thus, the mapping $J$ is the isometry and the algebras $H_{b \mathbb{P}}(X)$ and $H_{b \mathbb{P}}\left(\ell_{\infty}\right)$ are isometrically isomorphic.

Theorem 3. Let $P_{n}: \ell_{\infty} \rightarrow \mathbb{C}$ be defined by

$$
P_{n}(x)=x_{n}^{n}
$$

for $x=\left(x_{1}, x_{2}, \ldots\right) \in \ell_{\infty}$ and $\left\|P_{n}\right\|=1, n \in \mathbb{N}$. Then the spectrum $M_{b \mathbb{P}}$ of the algebra $H_{b \mathbb{P}}\left(\ell_{\infty}\right)$ coincides with the set of all point-evaluation functionals at points of $\ell_{\infty}$.

Proof. Let $\varphi \in M_{b \mathbb{P}}$ be a character that belongs to the spectrum of the algebra $H_{b \mathbb{P}}\left(\ell_{\infty}\right)$. Let us denote by $\delta_{x}$ the point-evaluation functional at a point $x \in \ell_{\infty}$. Let us show that $\varphi=\delta_{x}$ for some $x \in \ell_{\infty}$.

Every $\varphi \in M_{b \mathbb{P}}$ is uniquely determined by the sequence $\left(\varphi\left(P_{1}\right), \varphi\left(P_{2}\right), \ldots, \varphi\left(P_{n}\right), \ldots\right)$. Let us put $x=\left(\varphi\left(P_{1}\right), \sqrt[2]{\varphi\left(P_{2}\right)}, \ldots, \sqrt[n]{\varphi\left(P_{n}\right)}, \ldots\right)$. Since $\left\|P_{n}\right\|=1, n \in \mathbb{N}$, then according to the Proposition 2 the sequence $\left(\varphi\left(P_{1}\right), \varphi\left(P_{2}\right), \ldots, \varphi\left(P_{n}\right), \ldots\right)$ grows no faster than some geometric progression. Thus the sequence $x=\left(\varphi\left(P_{1}\right), \sqrt[2]{\varphi\left(P_{2}\right)}, \ldots, \sqrt[n]{\varphi\left(P_{n}\right)}, \ldots\right)$ is bounded and so, $x \in l_{\infty}$.

Besides, the following chain of equalities holds

$$
\delta_{x}\left(P_{n}\right)=P_{n}(x)=x_{n}^{n}=\left(\sqrt[n]{\varphi\left(P_{n}\right)}\right)^{n}=\varphi\left(P_{n}\right)
$$

Hence $\varphi=\delta_{x}$ and every character $\varphi \in M_{b \mathbb{P}}$ is a point-evaluation functional at some point of $\ell_{\infty}$.

Corollary 1. Let $c_{00} \subset X \subset \ell_{\infty}$ polynomials $P_{n}: X \rightarrow \mathbb{C}$ be defined by

$$
P_{n}(x)=x_{n}^{n}
$$

for every $x=\left(x_{1}, x_{2}, \ldots\right) \in X$. Then the spectrum $M_{b \mathbb{P}}$ of the algebra $H_{b \mathbb{P}}(X)$ coincides with the set of all point-evaluation functionals at points of $\ell_{\infty}$. 


\section{REFERENCES}

[1] Alencar R., Aron R.M., Galindo P., Zagorodnyuk A.V. Algebras of symmetric holomorphic functions on $\ell_{p}$. Bull. London Math. Soc. 2003, 35 (2), 55-64. doi:10.1112/S0024609302001431

[2] Aron R.M., Cole B.J., Gamelin T.W. Spectra of algebras of analytic functions on a Banach space. J. Reine Angew. Math. 1991, 415, 51-93.

[3] Aron R.M., Cole B.J., Gamelin T.W. Weak-star continuous analytic functions. Can. J. Math. 1995, 47, 673-683.

[4] Chernega I.V., Galindo P., Zagorodnyuk A.V. Some algebras of symmetric analytic functions and their spectra. Proc. Edinb. Math. Soc. 2012, 55, 125-142. doi:10.1017/S0013091509001655

[5] Galindo P., Vasylyshyn T.V., Zagorodnyuk A.V. Symmetric and finitely symmetric polynomials on the spaces $\ell_{\infty}$ and $L_{\infty}[0,+\infty]$. Math. Nachr. 2018, 291 (11-12), 1712-1726. doi:10.1002/mana.201700314

[6] Galindo P., Vasylyshyn T.V., Zagorodnyuk A.V. The algebra of symmetric analytic functions on $L_{\infty}$. Proc. Roy. Soc. Edinburgh 2017, 147 (4), 743-761. doi:10.1017/S0308210516000287

[7] Jawad F., Zagorodnyuk A.V. Supersymmetric Polynomials on the Space of Absolutely Convergent Series. Symmetry 2019, 11 (9), 1-16. doi:10.3390/sym11091111

[8] Kravtsiv V.V., Vasylyshyn T.V., Zagorodnyuk A.V. On Algebraic Basis of the Algebra of Symmetric Polynomials on $l_{p}\left(\mathbb{C}^{n}\right)$. J. Funct. Spaces 2017, 2017 (7), 8 p. doi:10.1155/2017/4947925

[9] Mujica J. Complex Analysis in Banach Spaces. North Holland 1986.

[10] Zagorodnyuk A.V. Spectra of Algebras of Entire Functions on Banach Spaces. Proc. Amer. Math. Soc. 2006, 134, 2559-2569. doi:10.1090/S0002-9939-06-08260-8

Received 10.09.2019

Галущак C.I. Спектри алгебр иілих функиій породжених деякою послідовністю поліномів // Карпатські матем. публ. - 2019. - Т.11, №2. - С. 311-320.

У даній роботі досліджено властивості топологічної алгебри цілих функцій, породженої зліченною множиною однорідних поліномів на комплексному банаховому просторі.

Нехай $X \in$ комплексним банаховим простором. Розглянуто підалгебру $H_{b \mathbb{P}}(X)$ алгебри Фреше цілих функцій обмеженого типу $H_{b}(X)$, породжену зліченною множиною алгебраїчно незалежних однорідних поліномів $\mathbb{P}$. Показано, що кожен член ряду Тейлора цілої функції, яка належить алгебрі $H_{b \mathbb{P}}(X), є$ алгебраїчною комбінацією елементів $\mathbb{P}$. Узагальнено теорему про обчислення радіус функції лінійного функціонала на випадок довільної підалгебри алгебри $H_{b}(X)$ на просторі $X$. Кожен неперервний лінійний мультиплікативний функціонал, який діє з $H_{b \mathbb{P}}(X)$ у $\mathbb{C}$ однозначно визначається послідовністю своїх значень на елементах $\mathbb{P}$. Як наслідок, існує взаємно однозначна відповідність між спектром (множиною всіх неперервних лінійних мультиплікативних функціоналів) алгебри $H_{b \mathbb{P}}(X)$ та деякою множиною послідовностей комплексних чисел. Встановлено оцінку зверху для послідовностей з цієї множини. Також доведено, що кожну функцію, яка належить алгебрі $H_{b \mathbb{P}}(X)$, де $X \in$ замкненим підпростором простору $\ell_{\infty}$ і містить простір $c_{00}$, можна єАиним чином аналітично продовжити на $\ell_{\infty}$ і алгебри $H_{b \mathbb{P}}(X)$ та $H_{b \mathbb{P}}\left(\ell_{\infty}\right) \in$ ізометрично ізоморфними. Описано спектр алгебри $H_{b \mathbb{P}}(X)$ у даному випадку для деякого спеціального виглялу елементів множини $\mathbb{P}$.

Результати даної роботи можуть бути використані для дослідження алгебри симетричних аналітичних функцій на банахових просторах.

Ключові слова і фрази: n-однорідний поліном, аналітична функція, спектр алгебри. 